Research Article

\title{
Option Pricing Formulas in a New Uncertain Mean-Reverting Stock Model with Floating Interest Rate
}

\author{
Zhaopeng Liu (iD) \\ School of Mathematics and Statistics, Suzhou University, Suzhou, Anhui 234000, China \\ Correspondence should be addressed to Zhaopeng Liu; lzp@ahszu.edu.cn
}

Received 16 June 2020; Revised 9 August 2020; Accepted 20 October 2020; Published 4 November 2020

Academic Editor: Filippo Cacace

Copyright (C) 2020 Zhaopeng Liu. This is an open access article distributed under the Creative Commons Attribution License, which permits unrestricted use, distribution, and reproduction in any medium, provided the original work is properly cited.

Options play a very important role in the financial market, and option pricing has become one of the focus issues discussed by the scholars. This paper proposes a new uncertain mean-reverting stock model with floating interest rate, where the interest rate is assumed to be the uncertain Cox-Ingersoll-Ross (CIR) model. The European option and American option pricing formulas are derived via the $\alpha$-path method. In addition, some mathematical properties of the uncertain option pricing formulas are discussed. Subsequently, several numerical examples are given to illustrate the effectiveness of the proposed model.

\section{Introduction}

Previous studies of option pricing are based on the assumption that the underlying asset price follows the stochastic differential equation [1-4]. According to the viewpoint of behavioral finance, the change of underlying asset price is not completely random. In fact, investors' belief degrees usually play an important role in real financial practice. So some scholars argued that stochastic differential equations may not be appropriate to describe the stock price process. Liu [5] founded a branch of axiomatic mathematics for modeling belief degrees. Liu [6] proposed the uncertain stock model and deduced the European option pricing formulas. Furthermore, the uncertainty theory is introduced into the financial field, then the uncertain financial theory is formed.

American option price formulas were derived by Chen [7]. Peng and Yao [8] proposed an uncertain stock model with mean-reverting process. Yao [9] gave the no-arbitrage determinant theorems on uncertain mean-reverting stock model in uncertain financial market. Zhang and Liu [10] investigated the pricing problem of geometric average Asian option. Yin et al. [11] gave the lookback option pricing formulas of uncertain exponential Ornstein-Uhlenbeck model, and Wang and Chen [12] derived Asian options pricing formulas in an uncertain stock model with floating interest rate. Zhang et al. [13] investigated the pricing problem of lookback options for uncertain financial market, and so on.

In this paper, we proposed a new uncertain stock model with floating interest rate. The European option and American option pricing formulas are investigated under the assumption that the underlying stock price follows an uncertain mean-reverting stock model, and the interest rate follows an uncertain CIR model.

\section{Preliminaries}

Uncertain measure $\mathscr{M}$ is a real-valued set function on a $\sigma$-algebra $\mathscr{L}$ over a nonempty set $\Gamma$ satisfying normality, duality, subadditivity, and product axioms [5].

Definition 1 (see [6]). An uncertain variable is a function $\xi$ from an uncertainty space $(\Gamma, \mathscr{L}, \mathscr{M})$ to the set of real numbers. The uncertainty distribution $\Phi$ of an uncertain variable $\xi$ follows as

$$
\Phi(x)=\mathscr{M}\{\xi \leq x\}
$$

for any real number $x$. If the uncertainty distribution $\Phi(x)$ is a continuous and strictly increasing function with respect to $x$ at which $0<\Phi(x)<1$, and 


$$
\begin{aligned}
& \lim _{x \longrightarrow-\infty} \Phi(x)=0, \\
& \lim _{x \longrightarrow+\infty} \Phi(x)=1,
\end{aligned}
$$

then $\Phi(x)$ is said to be a regular distribution, and the inverse function $\Phi^{-1}(\alpha)$ is called the inverse uncertainty distribution of $\xi$.

Definition 2 (see [14]). Let $\xi$ be an uncertain variable. Then the expected value of $\xi$ is defined by

$$
E[\xi]=\int_{0}^{+\infty} \mathscr{M}\{\xi \geq x\} \mathrm{d} x-\int_{-\infty}^{0} \mathscr{M}\{\xi \leq x\} \mathrm{d} x .
$$

provided that at least one of the two integrals is finite.

Theorem 1 (see [5]). Let $\xi$ be an uncertain variable with uncertainty distribution $\Phi$. If the expected value exists, then

$$
E[\xi]=\int_{0}^{+\infty}\{1-\Phi(x)\} \mathrm{d} x-\int_{-\infty}^{0} \Phi(x) \mathrm{d} x .
$$

Theorem 2 (see [14]). Let $\xi$ be an uncertain variable with regular uncertainty distribution $\Phi$. Then

$$
E[\xi]=\int_{0}^{1} \Phi^{-1}(\alpha) \mathrm{d} \alpha .
$$

Definition 3 (see [6]). The uncertain variables $\xi_{1}, \xi_{2}, \ldots, \xi_{n}$ are said to be independent if

$$
\mathscr{M}\left\{\bigcap_{i=1}^{n}\left(\xi_{i} \in B_{i}\right)\right\}=\bigwedge_{k=1}^{n} \mathscr{M}\left\{\xi_{i} \in B_{i}\right\} .
$$

for any Borel sets $B_{1}, B_{2}, \ldots, B_{n}$ of real numbers.

An uncertain process is a sequence of uncertain variables indexed by a totally ordered set $T$, which is used to model the evolution of uncertain phenomena.

Definition 4 (see [6]). An uncertain process $C_{t}$ is said to be a canonical Liu process if

(i) $C_{0}=0$ and almost all sample paths are Lipschitz continuous;

(ii) $C_{t}$ has stationary and independent increments;

(iii) every increment $C_{s+t}-C_{t}$ is a normal uncertain variable with expected value 0 and variance $t^{2}$, whose uncertainty distribution is

$$
\Phi(x)=\left(1+\exp \left(\frac{-\pi x}{\sqrt{3} t}\right)\right)^{-1} .
$$

Definition 5 (see [6]). Suppose $C_{t}$ is a canonical Liu process, $f$ and $g$ are two real functions. Then

$$
\mathrm{d} X_{t}=f\left(t, X_{t}\right) \mathrm{d} t+g\left(t, X_{t}\right) \mathrm{d} C_{t} .
$$

is called an uncertain differential equation with an initial value $X_{0}$.
Definition 6 (see [15]). Let $\alpha$ be a number with $0<\alpha<1$. An uncertain differential equation

$$
\mathrm{d} X_{t}=f\left(t, X_{t}\right) \mathrm{d} t+g\left(t, X_{t}\right) \mathrm{d} C_{t} .
$$

is said to have an $\alpha$-path $X_{t}^{\alpha}$ if it solves the corresponding ordinary differential equation

$$
\mathrm{d} X_{t}^{\alpha}=f\left(t, X_{t}^{\alpha}\right) \mathrm{d} t+\left|g\left(t, X_{t}^{\alpha}\right)\right| \Phi^{-1}(\alpha) \mathrm{d} t, \quad X_{0}^{\alpha}=X_{0},
$$

where $\Phi^{-1}(\alpha)$ is the inverse standard normal uncertainty distribution, i.e.,

$$
\Phi^{-1}(\alpha)=\frac{\sqrt{3}}{\pi} \ln \frac{\alpha}{1-\alpha} .
$$

Theorem 3 (see [15]). Assume that $X_{t}$ and $X_{t}^{\alpha}$ are the solution and $\alpha$-path of the uncertain differential equation

$$
\mathrm{d} X_{t}=f\left(t, X_{t}\right) \mathrm{d} t+g\left(t, X_{t}\right) \mathrm{d} C_{t},
$$

respectively. Then

$$
\begin{aligned}
& \mathscr{M}\left\{X_{t} \leq X_{t}^{\alpha}, \forall t\right\}=\alpha, \\
& \mathscr{M}\left\{X_{t}>X_{t}^{\alpha}, \forall t\right\}=1-\alpha .
\end{aligned}
$$

Theorem 4 (see $[15,16])$. Let $X_{t}$ and $X_{t}^{\alpha}$ be the solution and $\alpha$-path of the uncertain differential equation

$$
\mathrm{d} X_{t}=f\left(t, X_{t}\right) \mathrm{d} t+g\left(t, X_{t}\right) \mathrm{d} C_{t},
$$

respectively. Then, the solution $X_{t}$ has an inverse uncertainty distribution

$$
\Psi_{t}^{-1}(\alpha)=X_{t}^{\alpha}
$$

Theorem 5 (see [16]). Let $X_{t}$ and $X_{t}^{\alpha}$ be the solution and $\alpha$-path of the uncertain differential equation

$$
\mathrm{d} X_{t}=f\left(t, X_{t}\right) \mathrm{d} t+g\left(t, X_{t}\right) \mathrm{d} C_{t},
$$

respectively. Then, for any time $s>0$ and strictly increasing function $J(x)$, the supremum

$$
\sup _{0 \leq t \leq s} J\left(X_{t}\right)
$$

has an inverse uncertainty distribution

$$
\Psi_{s}^{-1}(\alpha)=\sup _{0 \leq t \leq s} J\left(X_{t}^{\alpha}\right)
$$

and the time integral $\int_{0}^{s} J\left(X_{t}\right) d t$ has an inverse uncertainty distribution

$$
\Psi_{s}^{-1}(\alpha)=\int_{0}^{s} J\left(X_{t}^{\alpha}\right) \mathrm{d} t
$$

Theorem 6 (see [16]). Let $X_{t}$ and $X_{t}^{\alpha}$ be the solution and $\alpha$-path of the uncertain differential equation 


$$
\mathrm{d} X_{t}=f\left(t, X_{t}\right) \mathrm{d} t+g\left(t, X_{t}\right) \mathrm{d} C_{t},
$$

respectively. Then, for any time $s>0$ and strictly decreasing function $J(x)$, the supremum

$$
\sup _{0 \leq t \leq s} J\left(X_{t}\right)
$$

has an inverse uncertainty distribution

$$
\Psi_{s}^{-1}(\alpha)=\sup _{0 \leq t \leq s} J\left(X_{t}^{1-\alpha}\right)
$$

and the time integral $\int_{0}^{s} J\left(X_{t}\right) d t$ has an inverse uncertainty distribution

$$
\Psi_{s}^{-1}(\alpha)=\int_{0}^{s} J\left(X_{t}^{1-\alpha}\right) \mathrm{d} t
$$

Liu [17] proposed that the uncertain processes $X_{1 t}, X_{2 t}, \ldots, X_{n t}$ are independent if, for any positive integer $k$ and any times $t_{1}, t_{2}, \ldots, t_{k}$, the uncertain vectors

$$
\xi_{i}=\left(X_{i t_{1}}, X_{i t_{2}}, \ldots, X_{i t_{k}}\right), \quad i=1,2, \ldots, n,
$$

are independent.

Theorem 7 (see [18]). Assume that $X_{1 t}, X_{2 t}, \ldots, X_{n t}$ are some independent uncertain processes derived from the solutions of some uncertain differential equations. If the function $f\left(x_{1}, x_{2}, \ldots, x_{n}\right)$ is strictly increasing with respect to $x_{1}, x_{2}, \ldots, x_{m}$ and strictly decreasing with respect to $x_{m+1}, x_{m+2}, \ldots, x_{n}$, then the uncertain process $X_{t}=f\left(X_{1 t}, X_{2 t}, \ldots, X_{n t}\right)$ has an $\alpha$-path

$$
X_{t}^{\alpha}=f\left(X_{1 t}^{\alpha}, \ldots, X_{m t}^{\alpha}, X_{m+1, t}^{1-\alpha}, \ldots, X_{n t}^{1-\alpha}\right) .
$$

\section{Uncertain Mean-Reverting Stock Model with Floating Interest Rate}

In the real market, the interest rate is an important economic indicator, which is always affected by some uncertain factors. To meet the needs of actual financial markets, Yao [18] assumed that both the interest rate $r_{t}$ and the stock price $X_{t}$ follow uncertain differential equations and presented an uncertain stock model with floating interest rate as follows,

$$
\left\{\begin{array}{l}
\mathrm{d} r_{t}=\mu_{1} r_{t} \mathrm{~d} t+\sigma_{1} r_{t} \mathrm{~d} C_{1 t} \\
\mathrm{~d} X_{t}=\mu_{2} X_{t} \mathrm{~d} t+\sigma_{2} X_{t} \mathrm{~d} C_{2 t}
\end{array}\right.
$$

where $\mu_{1}$ and $\sigma_{1}$ are the drift and diffusion of the interest rate, respectively, $\mu_{2}$ and $\sigma_{2}$ are the drift and diffusion of the stock price, respectively, and $C_{1 t}$ and $C_{2 t}$ are independent canonical Liu processes. Considering the long-term fluctuations of the stock price and the changing of the interest rate from over time, Sun and $\mathrm{Su}$ [19] proposed an uncertain mean-reverting stock model with floating interest rate to describe the stock price and interest rate.

$$
\left\{\begin{array}{l}
\mathrm{d} r_{t}=\left(m_{1}-a_{1} r_{t}\right) \mathrm{d} t+\sigma_{1} \mathrm{~d} C_{1 t} \\
\mathrm{~d} X_{t}=\left(m_{2}-a_{2} X_{t}\right) \mathrm{d} t+\sigma_{2} \mathrm{~d} C_{2 t}
\end{array}\right.
$$

In Sun and Su's model, the interest rate model was assumed to be the uncertain Vasicek model. There is no doubt that the Vasicek model could bring a negative value to the interest rate. However, the CIR model can overcome the problem, and it can ensure that the interest rate remains positive all the time.

In this paper, we will make some improvements to the stock models (27). To ensure that the interest rate is always positive, we assume the interest rate process to be the uncertain CIR model and introduce a new uncertain meanreverting stock model with floating interest rate,

$$
\left\{\begin{array}{l}
\mathrm{d} r_{t}=a_{1}\left(b_{1}-r_{t}\right) \mathrm{d} t+\sigma_{1} \sqrt{r_{t}} \mathrm{~d} C_{1 t}, \\
\mathrm{~d} X_{t}=\left(a_{2}-b_{2} X_{t}\right) \mathrm{d} t+\sigma_{2} \mathrm{~d} C_{2 t},
\end{array}\right.
$$

where $a_{1}$ represents the rate of adjustment of $r_{t}, b_{1}$ represents the average interest rate, $\sigma_{1}$ represents the interest rate diffusion, $a_{1}, b_{1}, \sigma_{1}, a_{2}, b_{2}, \sigma_{2}$ are constants, and $C_{1 t}$ and $C_{2 t}$ are independent canonical Liu processes.

\section{European Option Pricing Formulas}

4.1. European Call Option. A European call option offers the holder the right without the obligation to buy a certain asset at an expiration time $T$ with a strike price $K$, and $X_{t}$ is the stock price of the time $t$. The payoff of the European call option is given by $\left(X_{T}-K\right)^{+}$.

Definition 7. Assume European call option has a strike price $K$ and an expiration time $T$. Then the European call option price is

$$
f_{\mathrm{Ec}}=E\left[\exp \left(-\int_{0}^{T} r_{s} \mathrm{~d} s\right)\left(X_{T}-K\right)^{+}\right]
$$

Theorem 8 Assume European call option for the uncertain stock model (3) has a strike price $K$ and an expiration time $T$. Then the European call option price is

$$
f_{\mathrm{Ec}}=\int_{0}^{1} \exp \left(-\int_{0}^{T} r_{s}^{1-\alpha} \mathrm{d} s\right)\left(X_{T}^{\alpha}-K\right)^{+} \mathrm{d} \alpha,
$$

where $r_{s}^{1-\alpha}$ solves the following the ordinary differential equation

$$
\begin{aligned}
\mathrm{d} r_{s}^{1-\alpha}= & a_{1}\left(b_{1}-r_{s}^{1-\alpha}\right) \mathrm{d} s+\frac{\sqrt{3} \sigma_{1}}{\pi} \ln \frac{1-\alpha}{\alpha} \sqrt{r_{s}^{1-\alpha}} \mathrm{d} s \\
X_{T}^{\alpha}= & X_{0} \exp \left(-b_{2} T\right)+\left(1-\exp \left(-b_{2} T\right)\right) \\
& \cdot\left(\frac{a_{2}}{b_{2}}+\frac{\sigma_{2}}{b_{2}} \frac{\sqrt{3}}{\pi} \ln \frac{\alpha}{1-\alpha}\right) .
\end{aligned}
$$

Proof. According to Theorem 3, we can get the $\alpha$-path of the stock price $X_{t}$ :

$$
X_{t}^{\alpha}=X_{0} \exp \left(-b_{2} t\right)+\left(1-\exp \left(-b_{2} t\right)\right)\left(\frac{a_{2}}{b_{2}}+\frac{\sigma_{2}}{b_{2}} \frac{\sqrt{3}}{\pi} \ln \frac{\alpha}{1-\alpha}\right) \text {. }
$$


Similarly, we also get that $r_{s}^{1-\alpha}$ satisfies the differential equation,

$$
\mathrm{d} r_{s}^{1-\alpha}=a_{1}\left(b_{1}-r_{s}^{1-\alpha}\right) \mathrm{d} s+\frac{\sqrt{3} \sigma_{1}}{\pi} \ln \frac{1-\alpha}{\alpha} \sqrt{r_{s}^{1-\alpha}} \mathrm{d} s
$$
$\int_{0}^{T} r_{s}^{\alpha} \mathrm{d} s$

It follows from Theorem 5 that the $\alpha$-path of $\int_{0}^{T} r_{s} \mathrm{~d} s$ is

Since $y=\exp (-x)$ is strictly decreasing with respect to $x$, from Theorem 6 , the discount rate

$$
\exp \left(-\int_{0}^{T} r_{s} \mathrm{~d} s\right)
$$

has an $\alpha$-path

$$
\exp \left(-\int_{0}^{T} r_{s}^{1-\alpha} \mathrm{d} s\right)
$$

Since $\left(X_{T}-K\right)^{+}$is an increasing function with respect to $X_{T}$, it has an $\alpha$-path

$$
\begin{aligned}
\left(X_{T}^{\alpha}-K\right)^{+}= & \left(X_{0} \exp \left(-b_{2} T\right)+\left(1-\exp \left(-b_{2} T\right)\right)\right. \\
& \left.\cdot\left(\frac{a_{2}}{b_{2}}+\frac{\sigma_{2}}{b_{2}} \frac{\sqrt{3}}{\pi} \ln \frac{\alpha}{1-\alpha}\right)-K\right)^{+} .
\end{aligned}
$$

Therefore, the present value of the option

$$
\exp \left(-\int_{0}^{T} r_{s} \mathrm{~d} s\right)\left(X_{T}-K\right)^{+}
$$

has an $\alpha$-path

$$
\exp \left(-\int_{0}^{T} r_{s}^{1-\alpha} \mathrm{d} s\right)\left(X_{T}^{\alpha}-K\right)^{+}
$$

according to Theorem 7. We have the price of the European call option

$$
f_{\mathrm{Ec}}=\int_{0}^{1} \exp \left(-\int_{0}^{T} r_{s}^{1-\alpha} \mathrm{d} s\right)\left(X_{T}^{\alpha}-K\right)^{+} \mathrm{d} \alpha,
$$

according to Theorems 2 and 4 . The theorem is proved.

Theorem 9. Let $f_{E c}$ be the European call option price of the uncertain stock model (28). Then

(1) $f_{E c}$ is an increasing function of $X_{0}$;

(2) $f_{E c}$ is an increasing function of $a_{2}$;

(3) $f_{E c}$ is a decreasing function of $K$.

Proof. According to Theorem 8,

$$
\begin{aligned}
f_{\mathrm{Ec}}= & \int_{0}^{1} \exp \left(-\int_{0}^{T} r_{s}^{1-\alpha} \mathrm{d} s\right)\left(X_{0} \exp \left(-b_{2} T\right)\right. \\
& \left.+\left(1-\exp \left(-b_{2} T\right)\right)\left(\frac{a_{2}}{b_{2}}+\frac{\sigma_{2}}{b_{2}} \frac{\sqrt{3}}{\pi} \ln \frac{\alpha}{1-\alpha}\right)-K\right)^{+} \mathrm{d} \alpha,
\end{aligned}
$$

where $r_{s}^{1-\alpha}$ solves the following the ordinary differential equation

$$
\mathrm{d} r_{s}^{1-\alpha}=a_{1}\left(b_{1}-r_{s}^{1-\alpha}\right) \mathrm{d} s+\frac{\sqrt{3} \sigma_{1}}{\pi} \ln \frac{1-\alpha}{\alpha} \sqrt{r_{s}^{1-\alpha}} \mathrm{d} s
$$

(1) Since $\exp \left(-b_{2} T\right)>0$, the function $X_{0} \exp \left(-b_{2} T\right)>0$ is increasing with respect to $X_{0}$ and the European call option price $f_{\mathrm{Ec}}$ is increasing with respect to the initial stock price $X_{0}$. This means that the higher the initial stock price, the higher the European call option price.

(2) Since $1-\exp \left(-b_{2} T\right)>0, \quad b_{2}>0$, the function $1-\exp \left(-b_{2} T\right)\left(a_{2} / b_{2}\right)$ is increasing with respect to $a_{2}$ and the European call option price $f_{\mathrm{Ec}}$ is increasing with respect to the parameter $a_{2}$.

(3) Since the function

$$
\begin{gathered}
X_{0} \exp \left(-b_{2} T\right)+\left(1-\exp \left(-b_{2} T\right)\right) \\
\cdot\left(\frac{a_{2}}{b_{2}}+\frac{\sigma_{2}}{b_{2}} \frac{\sqrt{3}}{\pi} \ln \frac{\alpha}{1-\alpha}\right)-K,
\end{gathered}
$$

is decreasing with respect to $K$ and the European call option price $f_{\mathrm{Ec}}$ is decreasing with respect to the strike price $K$, this means that the higher the strike price, the lower the European call option price.

Example 1. Assume the parameters of the interest rate are $a_{1}=0.05, b_{1}=2, \sigma_{1}=0.04, r_{0}=0.03$, and the parameters of the stock price are $a_{2}=6, b_{2}=1, \sigma_{2}=3, X_{0}=4$, the strike price $K=5$ and the expiration time $T=1$. Then a European call option price is $f_{\mathrm{Ec}}=1.2859$.

4.2. European Put Option. Suppose that a European put option has a strike price $K$ and an expiration time $T$, and $X_{t}$ is the stock price of the time $t$. The payoff of the European put option is given by $\left(K-X_{T}\right)^{+}$.

Definition 8. Assume a European option has a strike price $K$ and an expiration time $T$. Then the European put option price is

$$
f_{\mathrm{Ep}}=E\left[\exp \left(-\int_{0}^{T} r_{s} \mathrm{~d} s\right)\left(K-X_{T}\right)^{+}\right] .
$$

Theorem 10. Assume a European option for the uncertain stock model (28) has a strike price $K$ and an expiration time $T$. Then the European put option price is

$$
f_{\mathrm{Ep}}=\int_{0}^{1} \exp \left(-\int_{0}^{T} r_{s}^{\alpha} \mathrm{d} s\right)\left(K-X_{T}^{\alpha}\right)^{+} \mathrm{d} \alpha,
$$

where $r_{s}^{\alpha}$ solves the following the ordinary differential equation: 


$$
\begin{aligned}
\mathrm{d} r_{s}^{\alpha}= & a_{1}\left(b_{1}-r_{s}^{\alpha}\right) \mathrm{d} s+\frac{\sqrt{3} \sigma_{1}}{\pi} \ln \frac{\alpha}{1-\alpha} \sqrt{r_{s}^{\alpha}} \mathrm{d} s, \\
X_{T}^{\alpha}= & X_{0} \exp \left(-b_{2} T\right)+\left(1-\exp \left(-b_{2} T\right)\right) \\
& \cdot\left(\frac{a_{2}}{b_{2}}+\frac{\sigma_{2}}{b_{2}} \frac{\sqrt{3}}{\pi} \ln \frac{\alpha}{1-\alpha}\right) .
\end{aligned}
$$

Proof. According to the proof of Theorem 8, we can get that the discount rate

$$
\exp \left(-\int_{0}^{T} r_{s} \mathrm{~d} s\right)
$$

has an $\alpha$-path

$$
\exp \left(-\int_{0}^{T} r_{s}^{1-\alpha} \mathrm{d} s\right)
$$

Since $\left(K-X_{T}\right)^{+}$is an decreasing function with respect to $X_{T}$, it has an $\alpha$-path

$$
\left(K-X_{T}^{1-\alpha}\right)^{+}=\left(K-\left(X_{0} \exp \left(-b_{2} T\right)+\left(1-\exp \left(-b_{2} T\right)\right)\left(\frac{a_{2}}{b_{2}}+\frac{\sigma_{2}}{b_{2}} \frac{\sqrt{3}}{\pi} \ln \frac{1-\alpha}{\alpha}\right)\right)\right)^{+} .
$$

Therefore, the present value of the option

$$
\exp \left(-\int_{0}^{T} r_{s} \mathrm{~d} s\right)\left(K-X_{T}\right)^{+}
$$

has an $\alpha$-path

$$
\exp \left(-\int_{0}^{T} r_{s}^{1-\alpha} \mathrm{d} s\right)\left(K-X_{T}^{1-\alpha}\right)^{+}
$$

according to Theorem 7 . We have the price of the European put option

$$
\begin{aligned}
f_{\mathrm{Ep}} & =\int_{0}^{1} \exp \left(-\int_{0}^{T} r_{s}^{1-\alpha} \mathrm{d} s\right)\left(K-X_{T}^{1-\alpha}\right)^{+} \mathrm{d} \alpha \\
& =\int_{0}^{1} \exp \left(-\int_{0}^{T} r_{s}^{\alpha} \mathrm{d} s\right)\left(K-X_{T}^{\alpha}\right)^{+} \mathrm{d} \alpha .
\end{aligned}
$$

According to Theorems 2 and 4. The theorem is proved.

Theorem 11. Let $f_{\text {Ep }}$ be the European put option price of the uncertain stock model (28). Then

(1) $f_{E p}$ is a decreasing function of $X_{0}$

(2) $f_{E p}$ is a decreasing function of $a_{2}$

(3) $f_{E p}$ is an increasing function of $K$

Proof. According to Theorem 10,

$$
\begin{aligned}
f_{\mathrm{Ep}}= & \int_{0}^{1} \exp \left(-\int_{0}^{T} r_{s}^{\alpha} \mathrm{d} s\right)\left(K-\left(X_{0} \exp \left(-b_{2} T\right)\right.\right. \\
& \left.\left.+\left(1-\exp \left(-b_{2} T\right)\right)\left(\frac{a_{2}}{b_{2}}+\frac{\sigma_{2}}{b_{2}} \frac{\sqrt{3}}{\pi} \ln \frac{\alpha}{1-\alpha}\right)\right)\right)^{+} \mathrm{d} \alpha .
\end{aligned}
$$

(1) Since $\exp \left(-b_{2} T\right)>0$, the function $-X_{0} \exp \left(-b_{2} T\right)$ is decreasing with respect to $X_{0}$ and the European put option price $f_{\mathrm{Ep}}$ is decreasing with respect to the initial stock price $X_{0}$. This means that the higher the initial stock price, the lower the European put option price.

(2) Since $1-\exp \left(-b_{2} T\right)>0, b_{2}>0$, the function $1-$ $\left(1-\exp \left(-b_{2} T\right)\right)\left(a_{2} / b_{2}\right)$ is decreasing with respect to $a_{2}$ and the European put option price $f_{\mathrm{Ep}}$ is decreasing with respect to the parameter $a_{2}$.

(3) Since the function

$$
K-\left(X_{0} \exp \left(-b_{2} T\right)+\left(1-\exp \left(-b_{2} T\right)\right)\left(\frac{a_{2}}{b_{2}}+\frac{\sigma_{2}}{b_{2}} \frac{\sqrt{3}}{\pi} \ln \frac{\alpha}{1-\alpha}\right)\right),
$$

is increasing with respect to $K$ and the European put option price $f_{\text {Ep }}$ is increasing with respect to the strike price $K$, this means that the higher the strike price, the higher the European put option price.

Example 2. Assume the parameters of the interest rate are $a_{1}=0.05, b_{1}=2, \sigma_{1}=0.04, r_{0}=0.03$, and the parameters of the stock price are $a_{2}=6, b_{2}=1, \sigma_{2}=3, X_{0}=4$, the strike price $K=5$ and the expiration time $T=1$. Then a European put option price is $f_{\mathrm{Ep}}=1.0362$.

\section{American Option Pricing Formulas}

5.1. American Call Option. The American call option gives the holder the right, without obligation, to buy an agreed quantity of stock at any time before the expiration date $T$ with a strike price $K$. Apparently, the best choice for the holder is to exercise the right at the supreme value, so the payoff of the American call option is given by $\sup \left(X_{t}-K\right)^{+}$. $0 \leq t \leq T$

Definition 9. Assume that the American call option has a strike price $K$ and an expiration time $T$. Then the American call option price is

$$
f_{\mathrm{Ac}}=E\left[\sup _{0 \leq t \leq T} \exp \left(-\int_{0}^{t} r_{s} \mathrm{~d} s\right)\left(X_{t}-K\right)^{+}\right] .
$$


Theorem 12. Assume that the American call option for the uncertain stock model (28) has a strike price $K$ and an expiration time $T$. Then the American call option price is

$$
f_{\mathrm{Ac}}=\int_{0}^{1} \sup _{0 \leq t \leq T} \exp \left(-\int_{0}^{t} r_{s}^{1-\alpha} \mathrm{d} s\right)\left(X_{t}^{\alpha}-K\right)^{+} \mathrm{d} \alpha,
$$

where $r_{s}^{1-\alpha}$ solves the following the ordinary differential equation:

$$
\begin{aligned}
\mathrm{d} r_{s}^{1-\alpha}= & a_{1}\left(b_{1}-r_{s}^{1-\alpha}\right) \mathrm{d} s+\frac{\sqrt{3} \sigma_{1}}{\pi} \ln \frac{1-\alpha}{\alpha} \sqrt{r_{s}^{1-\alpha}} \mathrm{d} s \\
X_{t}^{\alpha}= & X_{0} \exp \left(-b_{2} t\right)+\left(1-\exp \left(-b_{2} t\right)\right) \\
& \cdot\left(\frac{a_{2}}{b_{2}}+\frac{\sigma_{2}}{b_{2}} \frac{\sqrt{3}}{\pi} \ln \frac{\alpha}{1-\alpha}\right) .
\end{aligned}
$$

Proof. It follows from Theorem 8 that the uncertain process

$$
\exp \left(-\int_{0}^{t} r_{s} \mathrm{~d} s\right)\left(X_{t}-K\right)^{+},
$$

has an $\alpha$-path

$$
\exp \left(-\int_{0}^{t} r_{s}^{1-\alpha} \mathrm{d} s\right)\left(X_{t}^{\alpha}-K\right)^{+}
$$

According to Theorem 5, the uncertain process

$$
\sup _{0 \leq t \leq T} \exp \left(-\int_{0}^{t} r_{s} \mathrm{~d} s\right)\left(X_{t}-K\right)^{+},
$$

has an $\alpha$-path

$$
\sup _{0 \leq t \leq T} \exp \left(-\int_{0}^{t} r_{s}^{1-\alpha} \mathrm{d} s\right)\left(X_{t}^{\alpha}-K\right)^{+} .
$$

We have the price of the American call option

$$
f_{\mathrm{Ac}}=\int_{0}^{1} \sup _{0 \leq t \leq T} \exp \left(-\int_{0}^{t} r_{s}^{1-\alpha} \mathrm{d} s\right)\left(X_{t}^{\alpha}-K\right)^{+} \mathrm{d} \alpha .
$$

According to Theorems 2 and 4. The theorem is proved.

Theorem 13. Let $f_{A c}$ be the American call option price of the uncertain stock model (28). Then

(1) $f_{A c}$ is an increasing function of $X_{0}$

(2) $f_{A c}$ is an increasing function of $a_{2}$

(3) $f_{A c}$ is a decreasing function of $K$

Proof. According to Theorem 12,

$$
f_{\mathrm{Ac}}=\int_{0}^{1} \sup _{0 \leq t \leq T} \exp \left(-\int_{0}^{t} r_{s}^{1-\alpha} \mathrm{d} s\right)\left(X_{0} \exp \left(-b_{2} t\right)+\left(1-\exp \left(-b_{2} t\right)\right)\left(\frac{a_{2}}{b_{2}}+\frac{\sigma_{2}}{b_{2}} \frac{\sqrt{3}}{\pi} \ln \frac{\alpha}{1-\alpha}\right)-K\right)^{+} \mathrm{d} \alpha .
$$

(1) Since $\exp \left(-b_{2} t\right)>0$, the function $X_{0} \exp \left(-b_{2} t\right)>0$ is increasing with respect to $X_{0}$ and the American call option price $f_{\mathrm{Ac}}$ is increasing with respect to the initial stock price $X_{0}$. This means that the higher the initial stock price, the higher the American call option price.

(2) Since $1-\exp \left(-b_{2} t\right)>0, \quad b_{2}>0, \quad$ the function $\left(1-\exp \left(-b_{2} t\right)\right)\left(a_{2} / b_{2}\right)$ is increasing with respect to $a_{2}$ and the American call option price $f_{\mathrm{Ac}}$ is increasing with respect to the parameter $a_{2}$.

(3) Since the function

$$
X_{0} \exp \left(-b_{2} t\right)+\left(1-\exp \left(-b_{2} t\right)\right)\left(\frac{a_{2}}{b_{2}}+\frac{\sigma_{2}}{b_{2}} \frac{\sqrt{3}}{\pi} \ln \frac{\alpha}{1-\alpha}\right)-K,
$$

is decreasing with respect to $K$ and the American call option price $f_{\mathrm{Ac}}$ is decreasing with respect to the strike price $K$. This means that the higher the strike price, the lower the American call option price.

Example 3. Assume the parameters of the interest rate are $a_{1}=0.05, b_{1}=2, \sigma_{1}=0.04, r_{0}=0.03$, and the parameters of the stock price are $a_{2}=6, b_{2}=1, \sigma_{2}=3, X_{0}=4$, the strike price $K=5$ and the expiration time $T=1$. Then an American call option price is $f_{\mathrm{Ac}}=2.3762$.

5.2. American Put Option. The American put option gives the holder the right, without obligation, to sell an agreed quantity of stock at any time before the expiration date $T$ with a strike price $K$. Apparently, the best choice for the holder is to exercise the right at the supreme value, so the payoff of the American put option is given by $\sup _{0 \leq t \leq T}\left(K-X_{t}\right)^{+}$.

Definition 10. Assume that the American put option has a strike price $K$ and an expiration time $T$. Then the American put option price is

$$
f_{\text {Ap }}=E\left[\sup _{0 \leq t \leq T} \exp \left(-\int_{0}^{t} r_{s} \mathrm{~d} s\right)\left(K-X_{t}\right)^{+}\right] .
$$

Theorem 14. Assume that the American put option for the uncertain stock model (28) has a strike price $K$ and an expiration time T. Then the American put option price is 


$$
f_{\text {Ap }}=\int_{0}^{1} \sup _{0 \leq t \leq T} \exp \left(-\int_{0}^{t} r_{s}^{\alpha} \mathrm{d} s\right)\left(K-X_{t}^{\alpha}\right)^{+} \mathrm{d} \alpha,
$$

where $r_{s}^{\alpha}$ solves the following the ordinary differential equation:

$$
\begin{aligned}
\mathrm{d} r_{s}^{\alpha} & =a_{1}\left(b_{1}-r_{s}^{\alpha}\right) \mathrm{d} s+\frac{\sqrt{3} \sigma_{1}}{\pi} \ln \frac{\alpha}{1-\alpha} \sqrt{r_{s}^{\alpha}} \mathrm{d} s, \\
X_{t}^{\alpha} & =X_{0} \exp \left(-b_{2} t\right)+\left(1-\exp \left(-b_{2} t\right)\right)\left(\frac{a_{2}}{b_{2}}+\frac{\sigma_{2}}{b_{2}} \frac{\sqrt{3}}{\pi} \ln \frac{\alpha}{1-\alpha}\right) .
\end{aligned}
$$

Proof. It follows from Theorem 10 that the uncertain process

$$
\exp \left(-\int_{0}^{t} r_{s} \mathrm{~d} s\right)\left(K-X_{t}\right)^{+}
$$

has an $\alpha$-path

$$
\exp \left(-\int_{0}^{t} r_{s}^{1-\alpha} \mathrm{d} s\right)\left(K-X_{t}^{1-\alpha}\right)^{+} .
$$

According to Theorem 6, the uncertain process

$$
\sup _{0 \leq t \leq T} \exp \left(-\int_{0}^{t} r_{s} \mathrm{~d} s\right)\left(K-X_{t}\right)^{+},
$$

has an $\alpha$-path

$$
\sup _{0 \leq t \leq T} \exp \left(-\int_{0}^{t} r_{s}^{1-\alpha} \mathrm{d} s\right)\left(K-X_{t}^{1-\alpha}\right)^{+} .
$$

So, we have the price of the American put option

$$
\begin{aligned}
f_{\text {Ap }} & =\int_{0}^{1} \sup _{0 \leq t \leq T} \exp \left(-\int_{0}^{t} r_{s}^{1-\alpha} \mathrm{d} s\right)\left(K-X_{t}^{1-\alpha}\right)^{+} \mathrm{d} \alpha \\
& =\int_{0}^{1} \sup _{0 \leq t \leq T} \exp \left(-\int_{0}^{t} r_{s}^{\alpha} \mathrm{d} s\right)\left(K-X_{t}^{\alpha}\right)^{+} \mathrm{d} \alpha,
\end{aligned}
$$

according to Theorems 2 and 4 . The theorem is proved.

Theorem 15. Let $f_{A p}$ be the American put option price of the uncertain stock model (28). Then

(1) $f_{A p}$ is a decreasing function of $X_{0}$

(2) $f_{A p}$ is a decreasing function of $a_{2}$

(3) $f_{A p}$ is an increasing function of $K$

Proof. According to Theorem 14,

$$
f_{\mathrm{Ap}}=\int_{0}^{1} \sup _{0 \leq t \leq T} \exp \left(-\int_{0}^{t} r_{s}^{\alpha} \mathrm{d} s\right)\left(K-\left(X_{0} \exp \left(-b_{2} t\right)+\left(1-\exp \left(-b_{2} t\right)\right)\left(\frac{a_{2}}{b_{2}}+\frac{\sigma_{2}}{b_{2}} \frac{\sqrt{3}}{\pi} \ln \frac{\alpha}{1-\alpha}\right)\right)\right)^{+} \mathrm{d} \alpha,
$$

(1) Since $\exp \left(-b_{2} t\right)>0$, the function $-X_{0} \exp \left(-b_{2} t\right)<0$ is decreasing with respect to $X_{0}$ and the American put option price $f_{\mathrm{Ap}}$ is decreasing with respect to the initial stock price $X_{0}$. This means that the higher the initial stock price, the lower the American put option price.

(2) Since $1-\exp \left(-b_{2} t\right)>0, b_{2}>0$, the function $-(1-$ $\left.\exp \left(-b_{2} t\right)\right)\left(a_{2} / b_{2}\right)$ is decreasing with respect to $a_{2}$ and the American put option price $f_{\mathrm{Ap}}$ is decreasing with respect to the parameter $a_{2}$.

(3) Since the function

$$
K-\left(X_{0} \exp \left(-b_{2} t\right)+\left(1-\exp \left(-b_{2} t\right)\right)\left(\frac{a_{2}}{b_{2}}+\frac{\sigma_{2}}{b_{2}} \frac{\sqrt{3}}{\pi} \ln \frac{\alpha}{1-\alpha}\right)\right),
$$

is increasing with respect to $K$ and the American put option price $f_{\mathrm{Ap}}$ is increasing with respect to the strike price $K$, this means that the higher the strike price, the higher the American put option price.

Example 4. Assume that the parameters of the interest rate are $a_{1}=0.05, b_{1}=2, \sigma_{1}=0.04, r_{0}=0.03$, and the parameters of the stock price are $a_{2}=6, b_{2}=1, \sigma_{2}=3, X_{0}=4$, the strike price $K=5$ and the expiration time $T=1$. Then an American put option price is $f_{\text {Ap }}=1.6712$.

\section{Conclusions}

In this paper, we presented a new uncertain mean-reverting stock model with floating interest rate, in which the interest rate model was the uncertain CIR model. Based on the new model, we derived the pricing formulas of the European option and American option. In addition, some properties of the pricing formulas were also studied. Furthermore, some numerical examples were given. Further research could consider the lookback option pricing and power option pricing problems of the stock model.

\section{Data Availability}

The data used to support the findings of this study are included within the article.

\section{Conflicts of Interest}

The author declares that there are no conflicts of interest regarding the publication of this paper.

\section{Acknowledgments}

This research was supported by the Key Research Project of Humanities and Social Science of Anhui Province (SK2018A0472), the Nature Science Foundation of Anhui 
Provincial Education Department (KJ2017A443), and the Quality Engineering Project of Anhui Provincial Education Department (2016tszy083).

\section{References}

[1] F. Black and M. Scholes, "The pricing of options and corporate liabilities," Journal of Political Economy, vol. 81, no. 3, pp. 637-654, 1973.

[2] R. C. Heynen and H. M. Kat, "Lookback options with discrete and partial monitoring of the underlying price," Applied Mathematical Finance, vol. 2, no. 4, pp. 273-284, 1995.

[3] H. Y. Wong and Y. K. Kwok, "Sub-replication and replenishing premium: Efficient pricing of multi-state lookbacks," Review of Derivatives Research, vol. 6, no. 2, pp. 83-106, 2003.

[4] M. Dai, H. Y. Wong, and Y. K. Kwok, "Quanto lookback options," Mathematical Finance, vol. 14, no. 3, pp. 445-467, 2004.

[5] B. Liu, Uncertainty Theory, Springer, Berlin, Germany, 2007.

[6] B. Liu, "Some research problems in uncertainty theory," Journal of Uncertain Systems, vol. 3, no. 1, pp. 3-10, 2009.

[7] X. Chen, "American option pricing formula for uncertain financial market," International Journal of Operational Research, vol. 8, no. 2, pp. 32-37, 2011.

[8] J. Peng and K. Yao, "A new option pricing model for stocks in uncertainty markets," International Journal of Operational Research, vol. 8, no. 2, pp. 18-26, 2011.

[9] K. Yao, "No-arbitrage determinant theorems on meanreverting stock model in uncertain market," Knowledge-Based Systems, vol. 35, pp. 259-263, 2012.

[10] Z. Zhang and W. Liu, "Geometric average Asian option pricing for uncertain financial market," Journal of Uncertain Systems, vol. 8, no. 4, pp. 317-320, 2014.

[11] G. Yin, Y. Xiangfeng, and F. Zongfei, "Lookback option pricing problem of uncertain exponential Ornstein-Uhlenbeck model," Soft Computing, vol. 22, pp. 5647-5654, 2017.

[12] W. Wang and P. Chen, "Pricing asian options in an uncertain stock model with floating interest rate," International Journal for Uncertainty Quantification, vol. 8, no. 6, pp. 543-557, 2018.

[13] Z. Zhang, H. Ke, and W. Liu, "Lookback options pricing for uncertain financial market," Soft Computing, vol. 23, no. 14, pp. 5537-5546, 2019.

[14] B. Liu, Uncertainty Theory:a Branch of Mathematics for Modeling Human Uncertainty, Springer, Berlin, Germany, 2010.

[15] K. Yao and X. Chen, "A numerical method for solving uncertain differential equations," Journal of Intelligent \& Fuzzy Systems, vol. 25, no. 3, pp. 825-832, 2013.

[16] K. Yao, "Extreme values and integral of solution of uncertain differential equations," Journal of Uncertainty Analysis and Applications, vol. 1, no. 1, 2013.

[17] B. Liu, "Uncertainty distribution and independence of uncertain processes," Fuzzy Optimization and Decision Making, vol. 13, no. 3, pp. 259-271, 2014.

[18] K. Yao, "Uncertain contour process and its application in stock model with floating interest rate," Fuzzy Optimization and Decision Making, vol. 14, no. 4, pp. 399-424, 2015.

[19] Y. Sun and T. Su, "Mean-reverting stock model with floating interest rate in uncertain environment," Fuzzy Optimization and Decision Making, vol. 16, no. 2, pp. 235-255, 2017. 